\title{
The Spectrum of Idiopathic Ventricular Fibrillation and J-Wave Syndromes Novel Mapping Insights
}

\author{
Michel Haïssaguerre, $\mathrm{MD}^{\mathrm{a}, \mathrm{b}, \mathrm{c}, *}$, Wee Nademanee, $\mathrm{MD}^{\mathrm{d}}$, \\ Mélèze Hocini, MD ${ }^{a, b, c}$, Josselin Duchateau, PhD, MD ${ }^{a, b}$, \\ Clementine André, $\mathrm{MD}^{\mathrm{a}, \mathrm{b}}$, Thomas Lavergne, $\mathrm{MD}^{\mathrm{b}}$, Masa Takigawa, $\mathrm{MD}^{\mathrm{a}}$, \\ Frederic Sacher, MD ${ }^{a, b}$, Nicolas Derval, MD ${ }^{a, b}$, Thomas Pambrun, MD ${ }^{a, b}$, \\ Pierre Jais, $\mathrm{MD}^{\mathrm{a}, \mathrm{b}}$, Rick Walton, PhD ${ }^{\mathrm{b}}$, Mark Potse, PhD ${ }^{\mathrm{b}}$, Ed Vigmond, $\mathrm{PhD}^{\mathrm{b}}$, \\ Remi Dubois, $\mathrm{PhD}^{\mathrm{b}}$, Olivier Bernus, $\mathrm{PhD}^{\mathrm{b}, \mathrm{c}}$
}

\section{KEYWORDS}

- Sudden cardiac death $\bullet$ Ventricular fibrillation • Early repolarization syndromes

- J-wave syndromes • Purkinje system

\section{KEY POINTS}

- Idiopathic ventricular fibrillation (IVF) is defined as unexplained sudden cardiac death due to ventricular fibrillation (VF) without any identifiable structural or electrical cause after extensive investigations (no phenotype).

- Recent data show that the use of high-density electrophysiologic mapping may ultimately offer subclinical diagnoses of cardiac disease in about $90 \%$ of individuals with IVF. Two major conditions underlie the occurrence of VF: the presence of either depolarization abnormalities due to microstructural myocardial alteration or Purkinje abnormalities manifesting as triggering ectopy or reentry in the peripheral network.

- J-wave syndromes are defined as a distinct electrocardiographic phenotype (slurring/notch) affecting the junction between the QRS complex and the ST segment in inferolateral leads. Recent data provide evidence for heterogeneous substrates, related to either delayed depolarization due to microstructural alterations or early repolarization abnormalities.

- IVF and J-wave syndromes are the result of a wide spectrum of pathophysiologic processes. The individual phenotypic characterization is essential given its implications in therapy, genetic testing, and risk stratification.

Disclosure Statement: The authors have nothing to disclose.

This work was supported by the National Research Agency (ANR-10-IAHU04-LIRYC), the European Research Council (FP7/2007-2013 grant agreement number 322886-SYMPHONY), and the Leducq Foundation (RHYTHM network).

a Electrophysiology and Cardiac Stimulation, Bordeaux University Hospital, 311 President Wilson Boulevard, Bordeaux 33200, France; ${ }^{b}$ IHU LIRYC, Electrophysiology and Heart Modeling Institute, Avenue du Haut Leveque, Bordeaux 33604, Passes Cedex, France; ${ }^{c}$ Univ Bordeaux, CRCTB, U1045, Bordeaux, France; ${ }^{d}$ Bumrungrad Hospital, Bangkok 10110, Thailand

* Corresponding author. Institute of Rythmology and Heart Modelling, Hopital Cardiologique Haut-Leveque, Avenue de Magellan, Bordeaux-Pessac, France.

E-mail address: michel.haissaguerre@chu-bordeaux.fr 
Sudden cardiac death (SCD) remains a major health problem in all continents. Estimates vary around 350,000 victims per year in the United States or in Europe and are even higher in Southeast Asia. Coronary artery disease and cardiomyopathies are the main causes in older persons. ${ }^{1-4}$ However, in victims younger than 35 years, a common finding is the absence of structural heart disease (SHD) at autopsy, reported in $29 \%$ to $40 \%$ of cases particularly in young men. ${ }^{5-9}$ In the patients surviving after resuscitation maneuvers, ventricular fibrillation (VF) is consistently the lethal heart rhythm identified at the time of event. ${ }^{10,11}$ Over the last 20 years considerable efforts have been devoted to the search for discrete electrocardiographic or imaging signs and genetic markers in survivors. ${ }^{1,4,12}$ Despite this progress, unexplained SCD remains frequent in young adults.

The authors report here novel insights based on recent mapping data on 2 subsets: idiopathic VF defined as an idiopathic ventricular fibrillation (IVF) with no apparent structural or electrical heart disease after extensive investigations (eg, no phenotype) and $J$-wave syndromes defined as an electrocardiographic phenotype affecting the junction (J-wave) between the QRS complex and the ST segment in inferolateral leads.

\section{IDIOPATHIC VENTRICULAR FIBRILLATION}

SCD is commonly defined as death from an unexpected circulatory arrest, occurring within an hour of the onset of symptoms. Unexplained SCD is defined as no apparent structural or electrical heart disease after extensive investigations. When VF has been documented by electrocardiography during resuscitation maneuvers, unexplained SCD is termed as IVF. 5,13,14

\section{Criteria Defining Idiopathic Ventricular Fibrillation}

Based on published guidelines, patients classified as having IVF have no clinical evidence for drug abuse/intoxication or electrolyte abnormality at the time of initial presentation, no identifiable SHD demonstrated by normal echocardiographic and delayed gadolinium-enhanced MRI, no detectable coronary artery disease on coronary angiography or exercise testing, and no known repolarization abnormalities associated with long or short QT interval or J-wave syndromes. ${ }^{4,5,13}$ Pharmacologic testing with infusion of a sodiumchannel blocker (ajmaline, flecainide, or procainamide) is performed to exclude Brugada syndrome. Catecholamine infusion (isoprenaline and adrenaline) is performed to exclude catecholaminergic polymorphic ventricular tachycardia or arrhythmogenic right ventricular cardiomyopathy and to confirm the absence of long QT syndrome. Box 1 shows the flow diagram of diagnostic tests performed in patients referred for SCD.

According to the guidelines, genetic testing is recommended "for genetic arrhythmia syndromes in young patients ( $<40$ years of age) with IVF." The yield of genetic testing is higher if a family history of SCD at a young age is present. ${ }^{2,4}$ Familial screening is recommended in first-degree relatives and should include resting electrocardiogram (ECG), exercise testing, and echocardiography. In selected cases, Holter and signal-averaged ECGs, $\mathrm{MRI}$, and pharmacologic testing may be performed.

International protocols using whole exome sequencing are currently performed for improving the diagnosis yield of inherited disorders.

The absence of clinical phenotype defining a VF as idiopathic obviously depends on the resolution of investigations performed in each patient. The same reasoning also applies to the autopsy's results concluding for the absence of SHD. The authors have previously reported the ability of noninvasive mapping to identify the location of drivers (focal or reentrant) responsible for VF in man. ${ }^{9,15}$ This information has allowed to subsequently investigate the driver areas using high-density electrogram mapping and demonstrate the presence of microstructural cardiomyopathic alterations, which are a substrate of VF reentries.

The other chapters in this issue will emphasize the role of these microstructural myocardial alterations and that of Purkinje tissue in the pathophysiology of human IVF.

\section{Pathology of the Purkinje System in Idiopathic Ventricular Fibrillation}

The specialized Purkinje system constitutes a tiny part (about $2 \%$ ) of the myocardial mass, but its pathologic role in arrhythmogenesis is disproportionally high and pleomorphic. ${ }^{16,17}$ Purkinje pathology in IVF can manifest either as triggering ectopies or as arrhythmia maintenance via its complex network.

\section{Purkinje cells triggering idiopathic ventricular fibrillation}

When they can be identified in IVF (eg, in patients with frequent VF initiations), the great majority $(85 \%)$ of triggering ectopy originates from the Purkinje system or muscular structures associated with the Purkinje system, such as the moderator band and the papillary muscles. VF is usually triggered by short-coupled premature ventricular 


\begin{tabular}{l}
\hline Box 1 \\
Diagnostic tests performed in patients with \\
sudden cardiac death \\
Structural imaging \\
Including MRI (before ICD) \\
Electrocardiographic monitoring \\
Careful 12-lead documentation of ectopy \\
morphologies and couplings \\
Repolarization during cycle length variations \\
and dynamic maneuvers \\
Signal-averaged ECG \\
Stress test and pharmacologic tests \\
Na + blocker-adrenaline, isoprenaline \\
Electrophysiologic studies \\
High-density endoepicardial mapping \\
Programmed stimulation \\
EP-guided biopsy (optional) \\
Genetic testing \\
Abbreviations: ECG, electrocardiogram; EP, electro- \\
physiology; ICD, implantable cardioverter defibril- \\
lator; SCD, sudden cardiac death.
\end{tabular}

complexes (PVCs) but the authors have observed some patients with IVF in whom all documented Purkinje PVCs had long-coupling intervals greater than 360 ms (Fig. 1). The right ventricular outflow tract and less frequently the left ventricular outflow tract, or other myocardial locations are seen in $15 \%$ of cases, in the authors' current experience of 78 patients. The origin of ectopic trigger can be demonstrated by mapping the earliest origin (see Fig. 1) or by pace mapping if ectopies are rare. Many reports of successful ablation of triggering PVCs have been published, however, most of the time including only one or few cases. ${ }^{16}$ In the cases where runs of polymorphic PVCs have been reported, not only the initial one but also subsequent beats can be associated with Purkinje activity, suggesting repetitive reentry or triggered activity (see Fig. 1). This suggests that in addition to Purkinje firing, there is a potential to sustain activity within the Purkinje network, explaining the observations of successful outcome after ablation despite the persistence of isolated shortcoupled ectopy.

The ectopy morphology is essential to document on the 12-lead ECG as it guides mapping techniques by allowing focus on the area of interest. When originating from the left Purkinje fibers, the PVCs are narrow $(<120 \mathrm{~ms})$ with a right- bundle-branch block morphology. They demonstrate right or left axis deviation when originating from the anterior or the posterior Purkinje fibers, respectively. Morphology changes are frequently observed in the left Purkinje PVCs, indicating different exit sites or origins and thus a need for larger ablation. When originating from the right Purkinje arborization, the PVCs have a leftbundle-branch block morphology and left axis. ${ }^{16}$ They display a rapid initial deflection but have a wider QRS duration than left Purkinje PVCs, as the right Purkinje has a more limited spatial arborization. More discrete PVC morphology changes are observed, as compared with left Purkinje PVC.

In sinus rhythm, distal Purkinje potentials precede the QRS complex by less than or equal to $15 \mathrm{~ms}$. Longer intervals indicate a fascicular origin (with the risk of creating significant QRS changes if targeted for ablation). Special care should be taken during catheter manipulation to avoid inadvertent bumping of the right bundle, which will conceal Purkinje potentials within the local electrograms, the left-bundle-branch being much less vulnerable. When they are rare, PVCs can be inducible by creating postpacing (atrial or ventricular) pauses and/or drug infusion such as isoproterenol $(1-2 \mathrm{mcg} / \mathrm{kg} / \mathrm{min})$ or class I drugs.

\section{Repetitive reentry in the Purkinje network}

Reentry in the proximal Purkinje system has been described in the form of monomorphic ventricular tachycardia, either as bundle branch reentry or as left fascicular reentry. Polymorphic ventricular tachycardia has also been reported, with all QRS complexes being associated with Purkinje activity (see Fig. 1). The authors have observed 5 patients, survivors of IVF, who had neither short-coupling Purkinje ectopy nor microstructural myocardial alteration. A distinct electrophysiologic response was however observed during programmed stimulation at the distal left fascicular system.

No VF or repetitive activity was induced by stimulation from the right ventricle (in 4 of 5 patients), while pacing (S2-S3 extrastimuli) from the left posterior fascicle, and could consistently induce repetitive polymorphic ventricular tachycardia within the distal Purkinje system. The polymorphic QRS complexes were associated with distal Purkinje potentials on a beat-to-beat basis, whereas the proximal Purkinje fascicle and bundle branch potentials were slower or absent (excluding a bundle branch reentry). The variations in ventricular cycles were preceded by a similar change in Purkinje cycles; and arrhythmia termination was 

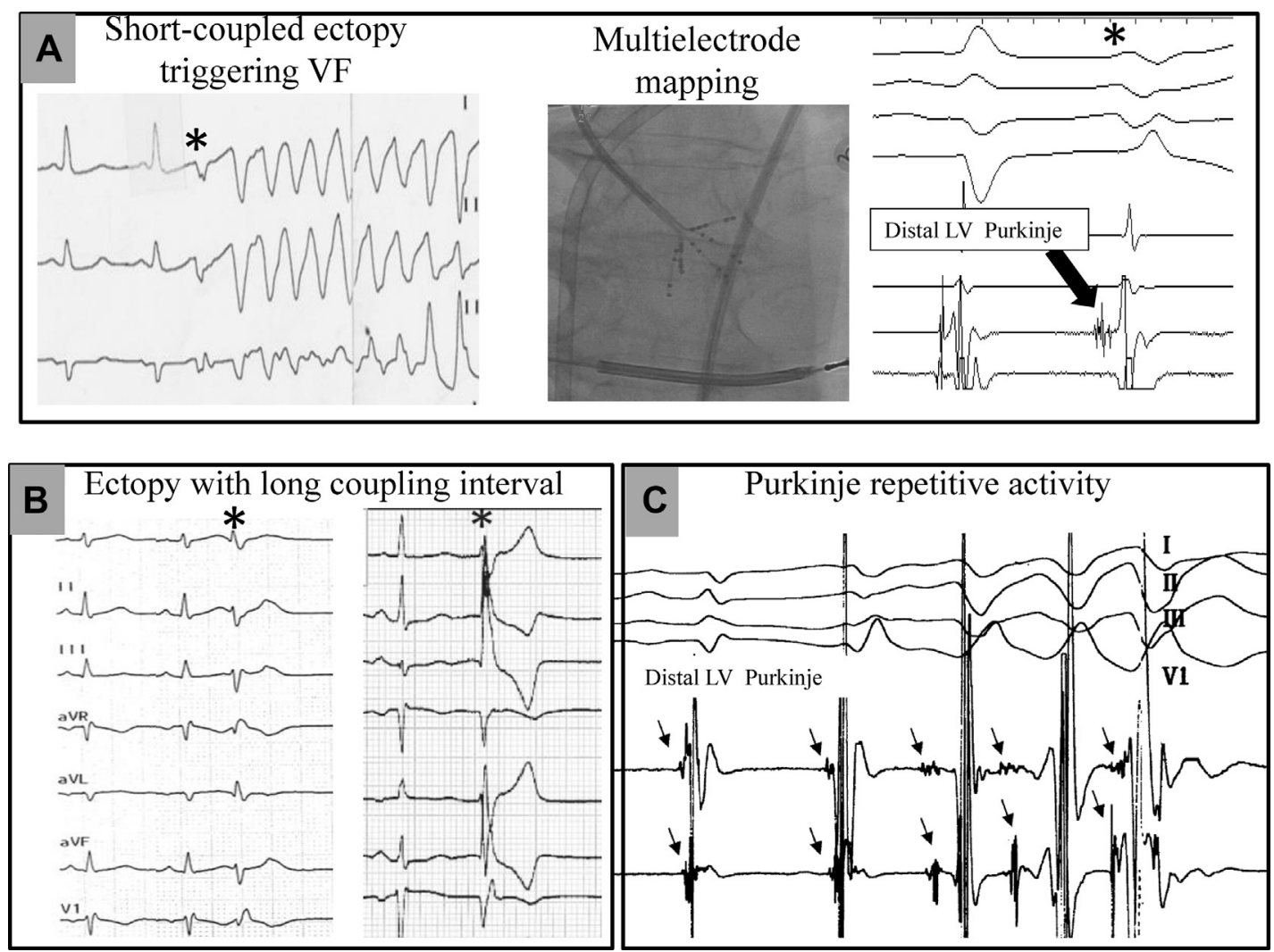

Fig. 1. Different patterns of Purkinje activity in 4 patients with IVF. (A) Typical short-coupled ectopy (asterisk) initiating VF. Multielectrode mapping with a multispline catheter (center) allows to record the earliest activity preceding ectopic beat in the distal left posterior fascicle (right). (B) Purkinje ectopy with long-coupling intervals (370 and $440 \mathrm{~ms}$ ) in 2 patients with IVF. (C) Spontaneous polymorphic ventricular tachycardia associated with 1to- 1 distal Purkinje activity. Note the varying Purkinje activation sequence between the distal and proximal bipoles that suggest changes in activation pathway. LV, left ventricle.

preceded by the disappearance of Purkinje activity (Fig. 2). Two of these cases have been published recently. ${ }^{18}$

These observations suggest that pacing performed near the distal left posterior Purkinje captures and invades retrogradely the Purkinje network. Although triggered activity cannot be ruled out, the repetitive responses are likely reentries induced in peripheral Purkinje system, with a gradual shift in trajectory and ventricular exit as demonstrated by computer modeling studies. These responses have been considered as clinically abnormal by the authors, as they are uncommon in their experience and have not been reported in the unique study evaluating left ventricular pacing in control patients. ${ }^{18}$ Further investigations are needed to confirm their pathologic significance and whether this electrophysiologic protocol may offer methods to reveal Purkinje abnormalities that can underlie VF susceptibility.

\section{MICROSTRUCTURAL MYOCARDIAL SUBSTRATE IN IDIOPATHIC VENTRICULAR FIBRILLATION}

In high-resolution experimental setups, VF requires the continuous formation of reentry for its maintenance, of which a critical determinant is the presence of normal (fiber arrangement) or abnormal (fibrosis) structural heterogeneities. In cardiomyopathic posttransplant human hearts, reentry has been shown to self-perpetuate for many cycles, as it can anchor to a myocardial scar. ${ }^{10,19}$ The authors have used multielectrode body surface recordings (ECGi) in clinics to identify the reentries during ongoing VF. Because patients with IVF are free of SHD, VF reentries were expected to be distributed homogeneously across both ventricles. However, a clustering of reentries was observed, and mapping of these regions in sinus rhythm revealed, in some of them, abnormal electrogram characteristics, indicating the presence of "microstructural" cardiomyopathic alteration. 


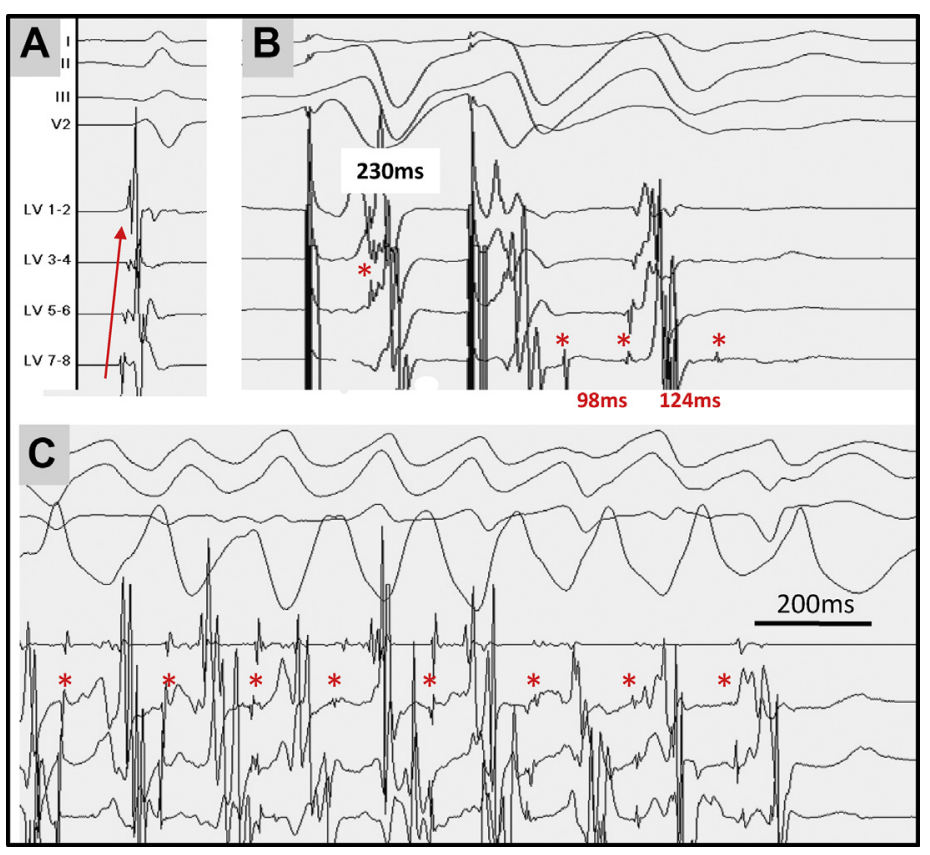

Fig. 2. Inducible repetitive activity in distal Purkinje. Recordings are performed along a decapolar catheter on the distal left posterior fascicle in sinus rhythm $(A)$ and during programmed stimulation $(B, C)$. Fig. $2 \mathrm{~B}$ shows double extrastimulation (230-230 ms), producing splitting of Purkinje (asterisks) activity (98 ms) followed by a third Purkinje activity 124 ms later: note the different involvement of parts of peripheral Purkinje system (LV7-8 vs LV 5-6). Fig. 2C shows double extrastimulation producing a polymorphic ventricular tachycardia in which each ventricular beat preceded by Purkinje activity, with varying sequence between recording bipoles. Arrhythmia termination is preceded by the disappearance of Purkinje activity. Repetitive Purkinje reentry was consistently reproducible and was the only electrophysiologic abnormality observed in this young woman surviving an episode of IVF. VT, ventricular tachycardia.

\section{Invasive Electrogram Mapping in Sinus Rhythm}

High-density recordings of endocardial and epicardial electrograms were performed using multispline catheter (Lasso or PentaRay catheter with $2 \mathrm{~mm}$ interelectrode distances) in the epicardium and PentaRay or decapolar catheters in the right and left endocardium. A transseptal or retroaortic approach was performed to access the endocardial left ventricle and a subxyphosternal approach to access into the pericardial space. The objective was to analyze electrograms present at the main driver areas (with the maximal number of rotations) compared with nondriver regions. Electroanatomical mapping was performed using magnetic geolocalization (CARTO system, Biosense Webster, CA); in a prior study, the authors mapped a mean of $590 \pm 403$ (endocardial right ventricle), $547 \pm 292$ (endocardial left ventricle), and $2081 \pm 1278$ (epicardium) sites per patient. Electrogram criteria were identical to those defining fibrotic and cardiomyopathic tissue during mapping of ischemic or dilated cardiomyopathies. Areas of low-amplitude electrograms were delineated in bipolar (less than $1 \mathrm{mV}$ ) and unipolar modes (less than 8.3 and $5.5 \mathrm{mV}$ in left and right ventricles respectively) on 3-dimensional ventricular reconstruction (CARTO system, BiosenseWebster,CA). Because low-amplitude electrograms can be due to normal fat tissue on the epicardium, epicardial electrograms were only considered abnormal if they harbored fragmented signals with a duration superior to 70 ms with more than 3 components or split/ late potentials. ${ }^{20-22}$ Greater mapping density was acquired in cases of abnormality to delineate the abnormal surface area.

\section{Results}

In the authors' current experience, myocardial areas manifesting low-amplitude and prolonged fractionated signals were found in 32 of 48 (67\%) patients. The abnormal tissue was clustered in 1 or 2 areas, whereas 4 patients had scattered abnormal areas. In a previous study the abnormal surface area covered a mean of $13 \pm 5 \mathrm{~cm}^{2}$, ranging from 6 to 22, representing $3.9 \pm 1.7 \%$ of the total ventricular surface. This finding is in keeping with prior experimental studies, which showed that even small ventricular lesions in the range of $4 \mathrm{~cm}^{2}$ are sufficient to promote VF. ${ }^{23,24}$

The right ventricle was the structure preferentially harboring the abnormal area, whereas the left ventricle or the septum was less frequently affected (Fig. 3). The comparison of endocardial and epicardial recordings at the same location showed that the abnormal signals were recorded in one side, mostly epicardial, indicating that the pathology involved a part of the ventricular wall rather than being transmural. 

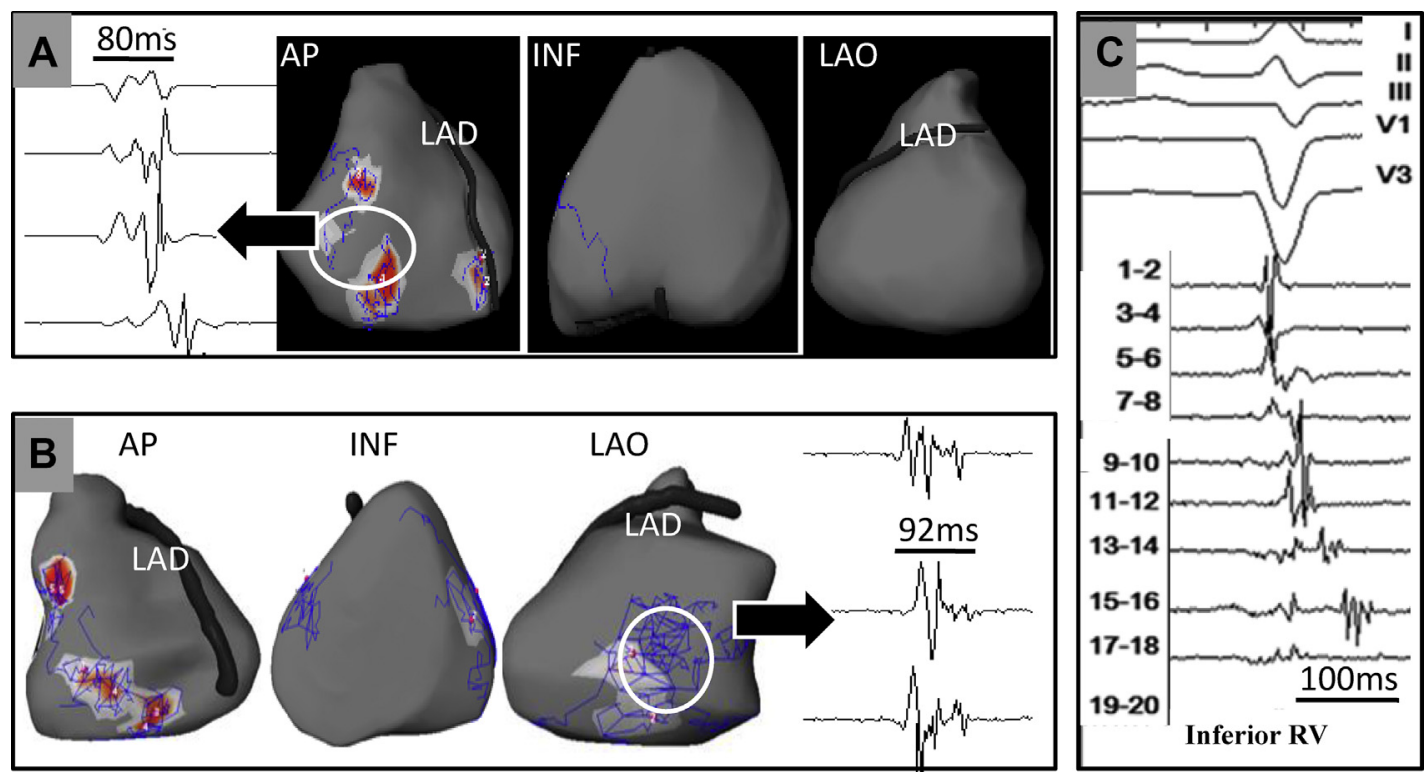

Fig. 3. Spatial location of abnormal electrogram areas in 3 patients with IVF. Two patients had spontaneous $(A)$ or induced $(B)$ sustained VF, with VF drivers mapped using body surface mapping. The trajectories of unstable and stable reentries are shown by the blue curves and red areas, respectively in 3 views, whereby the anterior view shows the right ventricle and the left anterior oblique view shows the left ventricle. The abnormal signals recorded in sinus rhythm during epicardial mapping are present within the white dotted contours, which collocated with a dominant area of VF reentries, in the epicardial right $(B)$ or left $(C)$ ventricle. All other ventricular regions show narrow signals indicating healthy underlying tissue. Case $C$ had no VF inducible by electrical stimulation. An area of prolonged and late electrograms was detected in the epicardial inferior right ventricle. AP, anteroposterior; LAD, left anterior descending; LAO, left anterior oblique; RV, right ventricle.

This aspect may explain why the abnormalities were unperceived by MRI. The reexamination of imaging data or retesting with ajmaline failed to identify structural abnormality or Brugada syndrome.

Among all abnormal areas, $86 \%$ colocalized within or at the border of a main driver region (see Fig. 3). This finding indicates that the mechanism of VF is linked to microstructural alterations acting as a substrate maintaining reentry, in the same way as overt structural heterogeneities maintain VF in SHDs.

\section{SPECTRUM OF IDIOPATHIC VENTRICULAR FIBRILLATION SUBSTRATES}

The phenotypic screening performed in 48 consecutive patients referred for IVF concluded that 11 patients $(23 \%)$ presented a Purkinje abnormality without microstructural myocardial abnormality, 32 patients $(67 \%)$ presented microstructural myocardial abnormalities, and 5 of them also having Purkinje triggers. Five patients had no apparent Purkinje or microstructural abnormality. Therefore, only $10 \%$ of IVF remained unexplained after comprehensive investigations. Unexplained SCD may result from an external ephemeris factor (as fever, vagal burst, hypokalemia, etc) necessary to reach a critical arrhythmogenic threshold and concealed causes such as long QT syndromes, Purkinje or microstructural abnormality etc., which may become detectable during follow-up or by genetic testing using genome wide sequencing (see Box 1).

The differentiation of IVF depending on Purkinje or myocardial dominant substrates has implications in terms of pathogenesis, diagnostic, genetics, and therapy. It is likely that both common and distinct diseases are involved in these different substrates and that a spectrum of different diseases such as genetic, inflammatory, or acquired cardiomyopathies can affect them. Improvements in imaging or electrophysiologic methods may be implemented in the future to identify the myocardial arrhythmogenic substrate before the occurrence of SCD. In terms of genetic predisposition, mutations in genes coding for specific Purkinje proteins versus genes coding for structural myocardial proteins may be differentiated as well as the interpretation of gene variants within the GWAS database, depending on the 
individual phenotype. Finally, in addition to Purkinje trigger ablation, the capability to identify localized structural alterations allows substrate ablation targeting the fragmented electrograms, such as in Brugada syndrome or SHDs.

\section{Inferolateral J-Wave Syndromes}

Early repolarization indicates a distinct electrocardiographic phenotype affecting the junction (J-wave) between the QRS complex and the ST segment in inferolateral leads. It was initially described as a benign ECG finding or in association with hypothermia. ${ }^{24}$ Subsequently, many conditions producing this phenotype have been described such as hypercalcemia, acute ischemia, brain injury, and others. ${ }^{25}$ The link with an increased risk of arrhythmic death was demonstrated in sporadic cases and in case-control studies of unexplained SCD then finally in association with various types of SHD. ${ }^{26-32}$ The later section reviews most recent mapping data in humans, which provide evidence for heterogeneity of substrates, which underlie inferolateral J-wave syndromes. For a comprehensive review of risk stratification and clinical management of $\mathrm{J}$-wave syndromes the readers are guided to other articles. $^{33-35}$

\section{Diagnosis of early repolarization and inferolateral J-wave syndromes}

Expert consensus recommendations ${ }^{33}$ defined the syndrome (the term indicating symptomatic patients) as follows: (1) the presence of J-point elevation greater than or equal to $1 \mathrm{~mm}$ in greater than or equal to 2 contiguous inferior and/or lateral leads of a standard 12-lead ECG in a patient resuscitated from otherwise unexplained VF; (2) an SCD victim with a negative autopsy and medical chart review with a previous ECG demonstrating $\mathrm{J}$-point elevation greater than or equal to $1 \mathrm{~mm}$ in greater than or equal to 2 contiguous inferior and/or lateral leads of a standard 12lead ECG. The ECG pattern (asymptomatic subjects) is defined by a distinct $\mathrm{J}$-wave or J-point elevation greater than or equal to $1 \mathrm{~mm}$ that is a notch or a slur, with or without ST-segment elevation.

This definition may be sometimes ambiguous owing to the small amplitude and spontaneous changes of the J-wave. Strong inspiration or Valsalva maneuvers can amplify $\mathrm{J}$-wave possibly by changing the heart shape and position relative to the chest. The dynamic changes in $\mathrm{J}$-waves during cycle-length prolongation, either unchanged pattern or amplification, are an important sign to evaluate, as it relates to the individual substrate underlying the $\mathrm{J}$-wave.

\section{High-density mapping of the J-wave}

In contrast to experimental wedge models focusing on repolarization abnormalities, ${ }^{31}$ recent high-density electrogram mapping in humans both in vivo and ex vivo experimental conditions provide evidence that a spectrum of heterogeneous substrates, related either to delayed depolarization or to early repolarization abnormalities, underlie inferolateral J-wave syndromes.

Clinical mapping data have been collected from patients with inferolateral $\mathrm{J}$-wave syndrome in 3 centers. ${ }^{36}$ These patients had no apparent SHD on MRI, and most were referred for VF recurring despite antiarrhythmic drugs including quinidine. A J-wave syndrome combined with a Brugada syndrome was observed in a subset, either spontaneously or with sodium channel blocker. Highdensity endocardial and epicardial (2000-6000 recorded points) electrograms were performed during sinus rhythm in bipolar and unipolar mode. A specific attention was paid to the electrograms coincident with the timing of $\mathrm{J}$-wave. The electrograms occurring within the J-wave were considered as belonging to depolarization if they were in temporal continuity of the surrounding depolarization field and sharp. They were considered as indicating ventricular repolarization if there was no continuity with surrounding depolarization electrograms and a slow pattern (hump) in unipolar mode. $^{37,38}$

J-wave due to delayed depolarization abnormality In patients referred with an isolated inferolateral J-wave syndrome, the J-wave was the expression of an area of delayed depolarization in the inferior part of ventricles in $15 \%$ to $30 \%$ of cases (depending on the inclusion center), whereas an early repolarization was the cause of $\mathrm{J}$-wave in the others $(70 \%-85 \%)$. The true prevalence of depolarization versus repolarization abnormality varied between the inclusion centers and was also biased as the patients could be referred after quinidine failure, which may have selected more patients with delayed depolarization. Delayed-depolarization $\mathrm{J}$-waves were recorded at the sites of terminal activation in the right ventricle or left ventricle. Most of the abnormal electrograms are found in epicardium, whereas 3 patients had abnormal electrograms recorded endocardially and epicardially. The terminology of early repolarization here is erroneous and "inferolateral J-wave" is more appropriate. An example of a late depolarization $\mathrm{J}$-wave is shown in Fig. 4.

In contrast, in the patients with combined J-wave and Brugada syndrome, J-wave was consistently caused by a delayed depolarization. 


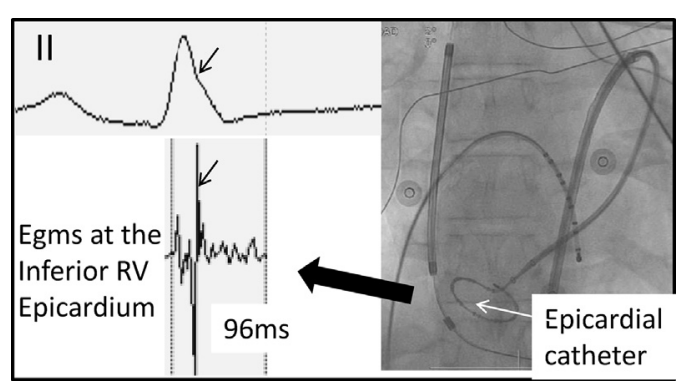

Fig. 4. A case of inferolateral J-wave syndrome due to abnormal depolarization. Epicardial depolarization in the inferobasal right ventricle occurs at the J-wave onset (small arrow) and is prolonged by low-voltage fragmented electrograms coincident with the J-wave body. Fluoroscopic anterior view shows the recording of circular 20-pole catheter in the epicardial inferobasal right ventricle and an endocardial decapolar catheter along the septum. ER, early repolarization.

The abnormal electrograms were recorded in inferior right ventricle and similar to those in the RVOT, suggesting structural alteration from the same pathogenesis. The absence of ST elevation in inferior leads, in contrast to V1-V2 leads, may be due to specific properties of the RVOT tissue, more prone to develop marked repolarization changes secondary to altered depolarization. ${ }^{39-42}$

Ajmaline testing was performed in all patients of the cohort and resulted in J-wave amplification or ST elevation in the inferior leads in only 5 patients. All 5 patients had a delayed depolarization, whereas no patient with early repolarization had $\mathrm{J} / \mathrm{ST}$ wave amplification on Ajmaline.

The results mentioned earlier are in keeping with the high prevalence of $\mathrm{J}$-waves described in various cardiomyopathies including noncompaction or arrhythmogenic right ventricular cardiomyopathies. In this context, it is likely that most of the J-waves express delayed depolarization.

J-wave due to early repolarization In this subset of patients there was no depolarization electrogram coincident with J-wave, but low-frequency signals (hump) were present particularly in unipolar recordings. Electrocardiographically, the increase of $\mathrm{J}$-waves during cycle-length prolongation was likely the most specific sign associated with early repolarization syndrome (Fig. 5).

The early repolarization potentials were dominantly located epicardially in the inferior septum and adjacent left ventricle including the regions of papillary muscles.

The $\mathrm{J}$-wave mechanism is likely here a voltage gradient across the ventricular wall during early phase of repolarization, as shown by Antzelevitch and Yan. ${ }^{31}$ However, the current clinical mapping

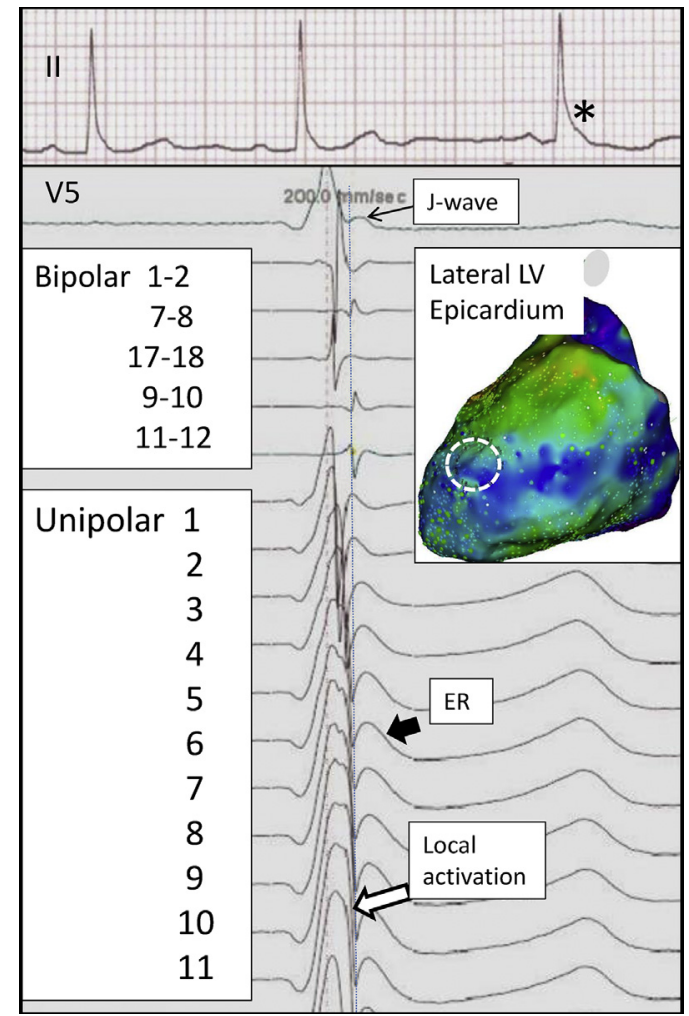

Fig. 5. A case of inferolateral J-wave syndrome due to early repolarization syndrome. The ECG shows J-wave amplification occurring in the third beat (asterisk) following a longer cycle length. Electrograms are recorded during epicardial mapping within the white dotted contour. Bipolar and unipolar recordings show the sharp local depolarization coincident with the very onset of J-wave (vertical line). The electrocardiographic J-wave is coincident with low-frequency ("ER") signals in unipolar recordings 1 to 11 , which cover the entire half-inferior surface of left ventricle in this patient.

techniques do not allow to measure repolarization parameters precisely and to demonstrate a gradient of repolarization or a phase 2 reentry. In patients with spontaneous VF initiation, the authors found an important triggering role of the Purkinje system further confirmed by favorable outcome after ablation (Fig. 6).

J-wave in human optical mapping In order to elucidate the substrates underlying J-wave syndrome, the authors' team had the unique opportunity to investigate human hearts with electrocardiographic J-wave patterns obtained through an organ donor program at the University Hospital. Epi- and endocardial optical mapping and microelectrode recordings were performed on either the left or the right ventricle. In one subject, the J-wave phenotype 


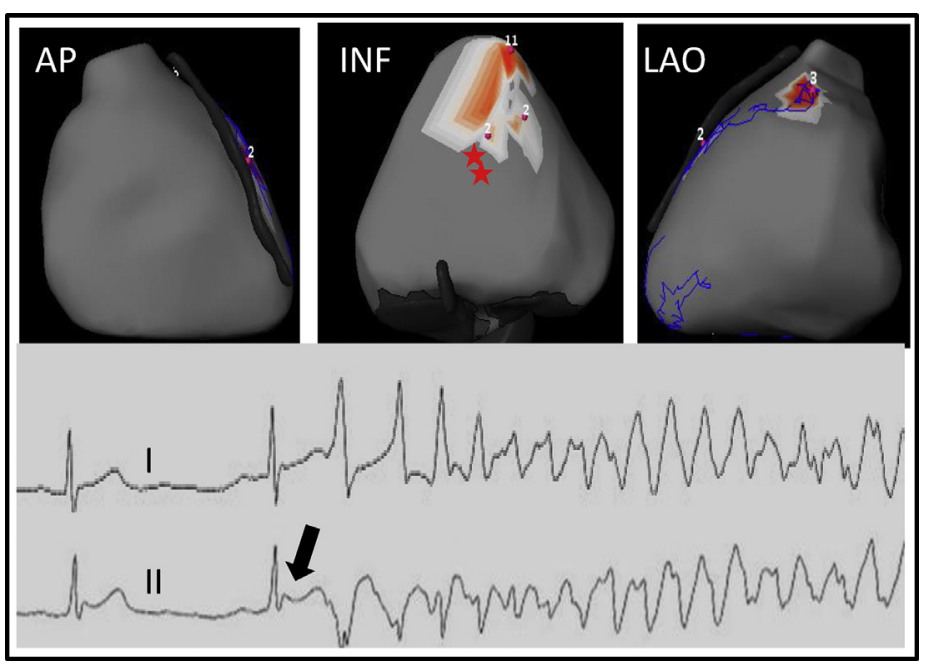

Fig. 6. Location of VF reentries in a 14-year-old patient with early repolarization (arrow). Noninvasive mapping is performed during the initial 2 seconds following spontaneous initiation of VF (ECG shown). The initial 2 beats seem as focal breakthroughs (2 red stars), whereas the subsequent 15 beats seem as reentries (in red) clustered in the inferior septal area (inferior view). The latter (epicardial) location is compatible with an exit from the (endocardial) left posterior Purkinje fascicle. INF, inferior.

was due to delayed conduction in the LV basal region with $80 \%$ activation time prolongation compared with control $(P<.05)$ and was exacerbated with increasing pacing frequency. For the other 2 subjects (who were siblings), J-waves increased during bradycardia and were associated with a heterogeneous increase of the action potential notch (phase 1 repolarization) and delayed action potential plateau on the right and/or left ventricular endocardium, but not epicardium, with no associated conduction abnormalities. These unique human heart data demonstrate that a variety of individual conduction and repolarization substrates, not limited to the epicardium, can underlie $\mathrm{J}$-wave syndromes in humans. ${ }^{15}$

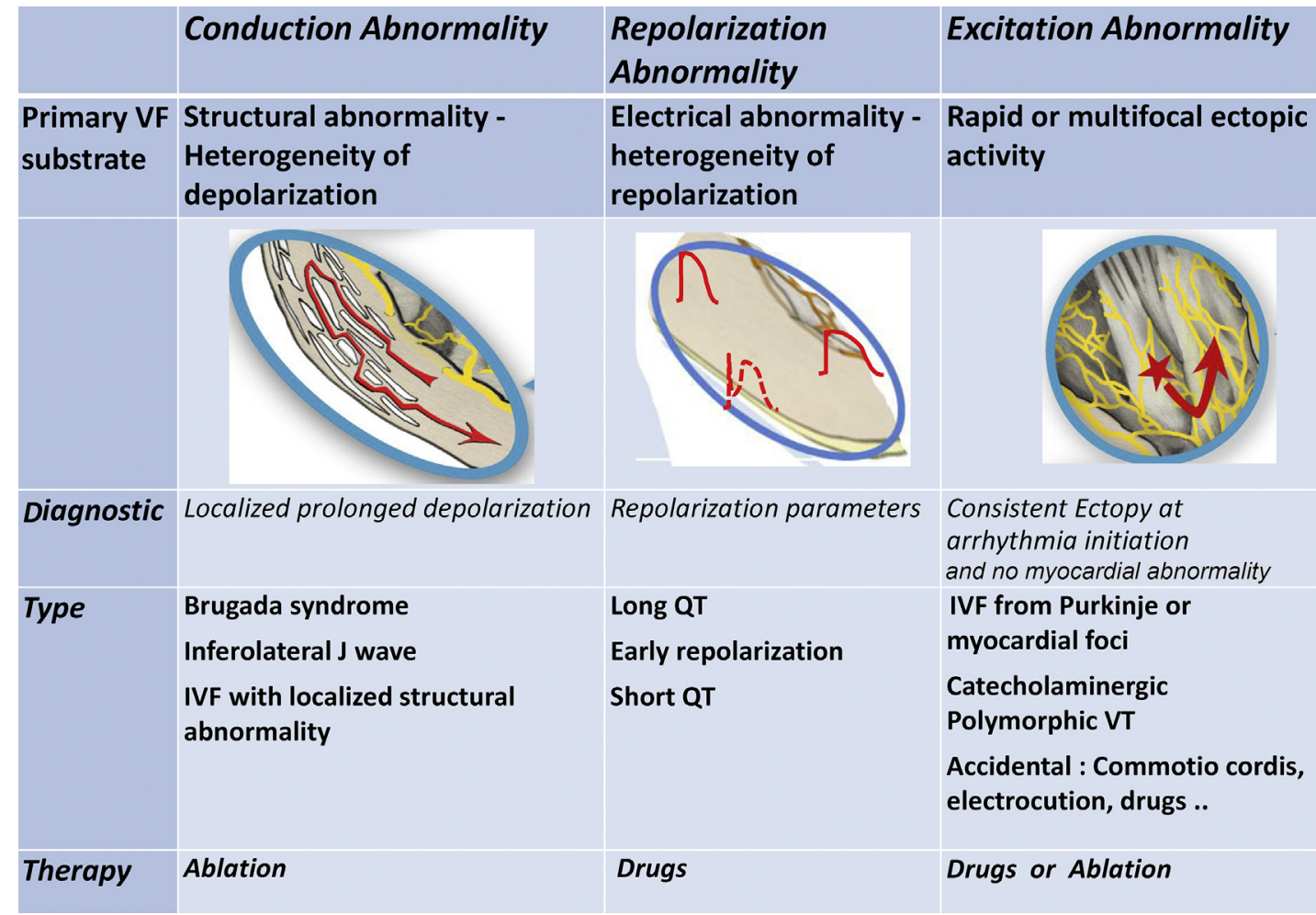

Fig. 7. The spectrum of arrhythmogenic diseases leading to SCD in apparently normal hearts, in 3 categories, based on the primary pathogenesis. 


\section{SUMMARY}

The present review shows that arrhythmogenic diseases leading to SCD in apparently normal hearts may be underlined by fundamentally different substrates despite similar ECG phenotypes (such as J-wave) or the absence of phenotype (IVF).

An important category is emerging in which the primary substrates maintaining VF are localized depolarization abnormalities due to microstructural myocardial alterations. Their pathogenesis is potentially of multiple causes, altering myocardial cells or their connections or leading to intervening fibrotic, inflammatory, or fatty tissue. A common feature of these substrates is that they constitute a target for efficient catheter ablation.

In a second category of SCD with structurally normal hearts, the primary VF substrates are repolarization abnormalities (long/short or early repolarization) for which drugs are the most appropriate therapy. In a third category the arrhythmia, mechanism seems dominated by "focal" activities from Purkinje or myocardial origin, such as catecholaminergic polymorphic ventricular tachycardia or a subset of IVF. This simplified classification (Fig. 7) does not exclude overlapping mechanisms. It puts together arrhythmogenic diseases, which are clinically distinct but share similar modes of pathogenesis and therapy, and potentially common genetic variants.

\section{REFERENCES}

1. Fishman GI, Chugh SS, Dimarco JP, et al. Sudden cardiac death prediction and prevention: report from a National Heart, Lung, and Blood Institute and Heart Rhythm Society Workshop. Circulation 2010;122:2335-48.

2. Priori SG, Blomström-Lundqvist C, Mazzanti A. 2015 ESC Guidelines for the management of patients with ventricular arrhythmias and the prevention of sudden cardiac death. Eur.Heart J 2015;36:2793-867.

3. Murakoshi N, Aonuma K. Epidemiology of arrhythmias and sudden cardiac death in Asia. Circ J 2013;77:2419-31

4. Al-Khatib SM, Stevenson WG, Ackerman MJ, et al. 2017 AHA/ACC/HRS guideline for management of patients with ventricular arrhythmias and the prevention of sudden cardiac death. Heart Rhythm 2018; 15(10):e190-252.

5. Visser M, van der Heijden JF, Doevendans PA, et al. Idiopathic ventricular fibrillation: the Struggle for definition, diagnosis, and follow-up. Circ Arrhythm Electrophysiol 2016;9:e003817.

6. Eckart R, Shry EA, Burke AP, et al. Sudden death in young adults. An autopsy-based series of a population undergoing active surveillance. J Am Coll Cardiol 2011;58:1254-61.

7. Winkel BG, Holst AG, Theilade J J, et al. Nationwide study of sudden cardiac death in persons aged 1-35 years. Eur Heart J 2011;32:983-90.

8. Bagnall RD, Weintraub RG, Ingles $J J$, et al. A prospective study of sudden cardiac death among children and young adults. N Engl J Med 2016:374:2441-52.

9. Haissaguerre M, Hocini M, Cheniti G, et al. Localized structural alterations underlying a subset of unexplained sudden cardiac death. Circ Arrhythm Electrophysiol 2018;11:e006120.

10. Gray RG, Pertsov AM, Jalife J. Spatial and temporal organization during cardiac fibrillation. Nature 1998; 392:75-8.

11. Nash MP, Mourad A, Clayton RH, et al. Evidence for multiple mechanisms in human ventricular fibrillation. Circulation 2016;114:536-42.

12. Torkamani A A, Muse ED, Spencer EG, et al. Molecular autopsy for sudden unexpected death. JAMA 2016;316:1492-4.

13. Krahn AD, Healey JS, Chauhan V. Systematic assessment of patients with unexplained cardiac arrest: cardiac arrest survivors with preserved ejection fraction registry (CASPER). Circulation 2009;120: 278-85.

14. Viskin S, Lesh MD, Eldar M, et al. Mode of onset of malignant ventricular arrhythmias in idiopathic ventricular fibrillation. J Cardiovasc Electrophysiol 1997;8(10):1115-20.

15. Haissaguerre M, Vigmond E, Stuyvers B, et al. Ventricular arrhythmias and the His-Purkinje system. Nat Rev Cardiol 2016;13:155-66.

16. Wilde AAM, Garan H, Boyden PA. Role of the Purkinje system in heritable arrhythmias. Heart Rhythm 2019;16:1121-6.

17. Haissaguerre M, Cheniti G, Escande W, et al. Idiopathic ventricular fibrillation associated with repetitive activity inducible within the distal Purkinje system. Heart Rhythm 2019. https://doi.org/10.1016/j.hrthm. 2019.04.012 [pii:S1547-5271(19)30312-1].

18. Nair K, Umapathy K, Farid T. Intramural activation during early human ventricular fibrillation. Circ Arrhythm Electrophysiol 2011;4:692-703.

19. Hsia H, Callans DJ, Marchlinski FE. Characterization of endocardial electrophysiological substrate in patients with nonischemic cardiomyopathy and monomorphic ventricular tachycardia. Circulation 2003; 108:704-10.

20. Soejima K, Stevenson WG, Sapp JL, et al. Endocardial and epicardial radiofrequency ablation of ventricular tachycardia associated with dilated cardiomyopathy: the importance of low-voltage scars. J Am Coll Cardiol 2004; 43:1834-41.

21. Cano O, Hutchinson M, Lin D. Electroanatomic substrate and ablation outcome for suspected 
epicardial left ventricular tachycardia in nonischemic cardiomyopathy. J Am Coll Cardiol 2009; 54:799-808.

22. Janse MJ, Kléber AG. Electrophysiological changes and ventricular arrhythmias in the early phase of regional myocardial ischemia. Circ Res 1981;49: 1069-81.

23. Kubota I, Lux RL, Burgess MJ, et al. Activation sequence at the onset of arrhythmias induced by localized myocardial warming and programmed premature stimulation in dogs. J Electrocardiol 1988;21:345-54.

24. Tomaszewski W. Changements electrocardiographiques observes chez un homme mort de froid. Arch Mal Cœur 1938;31:525-8.

25. Gussak I, Antzelevitch C. Early repolarization syndrome: clinical characteristics and possible cellular and ionic mechanisms. J Electrocardiol 2000;33: 299-309.

26. Otto CM, Tauxe RV, Cobb LA, et al. Ventricular fibrillation causes sudden death in southeast Asian immigrants. Ann Intern Med 1984;101:45-7.

27. Aizawa Y, Tamura M, Chinushi M, et al. Idiopathic ventricular fibrillation and bradycardia-dependent intraventricular block. Am Heart J 1993;126:1473-4.

28. Haissaguerre M, Derval N, Sacher F, et al. Sudden cardiac arrest associated with early repolarization. N Engl J Med 2008;358:2016-23.

29. Tikkanen JT, Anttonen O, Junttila MJ, et al. Longterm outcome associated with early repolarization on electrocardiography. N Engl J Med 2009;361: 2529-37.

30. Rosso R, Kogan E, Belhassen B, et al. J-point elevation in survivors of primary ventricular fibrillation and matched control subjects: incidence and clinical significance. J Am Coll Cardiol 2008;52:1231-8.

31. Antzelevitch C, Yan GX. J-wave syndromes: Brugada and early repolarization syndromes. Heart Rhythm 2015;12:1852-66.

32. Macfarlane PW, Antzelevitch C, Haissaguerre M, et al. The early repolarization pattern: a consensus paper. J Am Coll Cardiol 2015;66:470-7.
33. Viskin S, Rosso R, Halkin A. Making sense of early repolarization. Heart Rhythm 2012;9:566-9.

34. Obeyesekere N, Krahn AD. Early repolarization what should the clinician do? Arrhythm Electrophysiol Rev 2015;4:96-9.

35. Nademanee K, Haissaguerre M, Hocini $M$, et al. Ventricular Fibrillation substrates and Electrophysiological Abnormalities In Early Repolarization Syndrome: A Tale Of Two Phenotypes. HRS San francisco. Heart Rhythm Scientific Sessions S-P001-131. 2019.

36. Haissaguerre M, Nademanee W, Hocini M, et al. Depolarization versus repolarization abnormality underlying inferolateral $J$ wave syndromes-new concepts in sudden cardiac death with apparently normal hearts. Heart Rhythm 2018;16(5):781-90.

37. Boineau J. The early repolarization variant-an electrocardiographic enigma with both QRS and J-STT anomalies. J Electrocardiol 2007;40:3.e1-10.

38. Coronel R, Casini S, Koopmann TT, et al. Right ventricular fibrosis and conduction delay in a patient with clinical signs of Brugada syndrome: a combined electrophysiological, genetic, histopathologic, and computational study. Circulation 2005;112: 2769-77.

39. Martini M, Nava A, Thiene G, et al. Ventricular fibrillation without apparent structural heart disease. Am Heart J 1989;118:1203-9.

40. Hoogendijk MG, Potse M, Linnenbank AC, et al. Mechanism of right precordial ST-segment elevation in structural heart disease: excitation failure by current-to-load mismatch. Heart Rhythm 2010;7: 238-48.

41. Benoist D, Charron S, Dubes VN, et al. Arrhythmogenic Molecular Substrate In The Healthy Right Ventricular Outflow Tract Heart Rhythm Scientific Sessions C-P001-04. 2017.

42. Bernus $O$, Walton RD, Hof T, et al. A Wide Spectrum Of Substrates Underlie J-wave Syndromes In Humans. HRS chicago. Heart Rhythm Scientific Sessions S-MP13-05. 2019. 